\title{
Clinical Outcomes of Patients Treated for Candida auris Infections in a Multisite Health System, Illinois, USA
}

\author{
Kellie Arensman, Jessica L. Miller, Anthony Chiang, Nathan Mai, Joseph Levato, \\ Erik LaChance, Morgan Anderson, Maya Beganovic, Jennifer Dela Pena
}

Candida auris is an emerging fungal pathogen that is typically resistant to fluconazole and is known to cause healthcare-associated outbreaks. We retrospectively reviewed 28 patients who had $\geq 1$ positive culture for $C$. auris within a multisite health system in Illinois, USA, during May 2018-April 2019. Twelve of these patients were treated as inpatients for C. auris infections; 10 (83\%) met criteria for clinical success, defined as absence of allcause mortality, $C$. auris recurrence, and infection-related readmission at 30 days from the first positive culture. The other 2 patients (17\%) died within 30 days. Most patients $(92 \%)$ were empirically treated with micafungin. Four $(14 \%)$ of 28 total isolates were resistant to fluconazole, $1(3.6 \%)$ was resistant to amphotericin B, and 1 $(3.6 \%)$ was resistant to echinocandins. Our findings describe low rates of antifungal resistance and favorable clinical outcomes for most $C$. auris patients.

Candida auris is an emerging, multidrug-resistant, healthcare-associated fungal pathogen that was first reported in Japan in 2009 and has now been isolated on 6 continents (1-9). C. auris has been identified as the causative pathogen in various invasive fungal infections, including bloodstream infections $(2,4)$, and is associated with outbreaks across healthcare settings $(6,10)$. Risk factors for $C$. auris infection are similar to other Candida infections including

Author affiliations: Advocate Lutheran General Hospital, Park Ridge, Illinois, USA (K. Arensman, M. Beganovic, J. Dela Pena); Advocate South Suburban Hospital, Hazel Crest, Illinois, USA (J.L. Miller); Advocate Trinity Hospital, Chicago, Illinois, USA (J.L. Miller); Swedish Covenant Hospital, Chicago (A. Chiang); Advocate Christ Medical Center, Oak Lawn, Illinois, USA (N. Mai, J. Levato); Advocate Illinois Masonic Medical Center, Chicago (E. LaChance); Advocate Condell Medical Center, Libertyville, Illinois, USA (M. Anderson); Advocate Good Shepherd Hospital, Barrington, Illinois, USA (M. Anderson)

DOI: https://doi.org/10.3201/eid2605.191588 prolonged hospitalization, abdominal surgery, diabetes mellitus, intensive care unit (ICU) admission, use of central venous and urinary catheters, immunocompromising conditions, chronic kidney disease, and exposure to broad-spectrum antibiotic and antifungal agents (10-13). Investigations in the Chicago, Illinois, USA, area have found a high prevalence of $C$. auris colonization at ventilator-capable skilled nursing facilities (14) and have shown higher rates of $C$. auris colonization among patients who are mechanically ventilated, have a gastrostomy tube, or have a urinary catheter (15). Reported mortality rates attributable to invasive C. auris infection range from $30 \%$ to $59 \%$ globally $(13,16)$ and from $22 \%$ to $57 \%$ in the United States $(8,10,17)$.

C. auris isolates are often resistant to fluconazole and have variable susceptibility to other antifungal agents $(13,16)$. The Centers for Disease Control and Prevention (CDC) currently recommends echinocandins as empiric therapy for suspected or confirmed C. auris infections (18). However, recent reports have indicated reduced susceptibilities to echinocandins and suggest that resistance might be inducible under antifungal pressure $(8,16)$.

Previous reports of $C$. auris infections and outbreaks have largely focused on epidemiologic information, and data on treatment strategies and clinical outcomes are limited $(6,8,10,16-21)$. We report microbiologic data for $C$. auris isolates from a multisite health system in Illinois and an assessment of clinical outcomes for patients treated for $C$. auris infections.

\section{Methods}

This study is a retrospective cohort analysis of patients at 8 hospitals within a single health system located in the Chicago metropolitan area. We included all patients $\geq 18$ years old who had $\geq 1$ positive culture for $C$. auris from any anatomic site during January 1 , 
2008-April 30, 2019; we excluded pregnant patients, prisoners, and patients $<18$ years of age. If a patient had multiple positive cultures for $C$. auris, we included only the first positive culture per hospital encounter. Patients who died before culture result were not included in clinical success evaluation. The study received a non-human subjects research determination from the Advocate Aurora Health Institutional Review Board.

The microbiology laboratory for this system primarily uses matrix-assisted laser desorption/ionization time-of-flight (MALDI-TOF) mass spectrometry (Vitek MS, bioMérieux, https://www.biomerieux. com) for organism identification. Because our database does not include $C$. auris, for isolates not identified by our MALDI-TOF mass spectrometry system, we used Vitek 2 version 8.01 (bioMérieux), which we have been using since December 2017. We sent isolates identified as C. haemulonii, C. duobushaemulonii, and Candida spp. not identified by Vitek 2 to the Illinois Department of Public Health for additional testing using MALDI-TOF mass spectrometry or genomic sequencing to rule out misidentification of $C$. auris. During June 2018-April 2019, we sent all isolates identified as C. auris to the Illinois Department of Public Health, which forwarded them to CDC for whole-genome sequencing. We performed antifungal susceptibility testing with colorimetric microdilution by using Sensititer YeastOne YO9 (TREK Diagnostic Systems, https://www.trekds.com). Because no C. auris susceptibility breakpoints have been established, we used tentative breakpoints published by CDC for interpretation in this study (22).

We performed manual chart review for all patients. We evaluated patient charts for demographic information, infection source, culture source and susceptibilities, empiric and definitive therapy, length of hospital and ICU stay, clinical success, and reports of adverse events associated with treatment for $C$. auris infection. We defined clinical success as the absence of 30-day all-cause mortality, 30-day recurrence of the same organism, and 30-day infection-related readmission. We identified adverse drug events associated with antifungal therapy by reviewing patient laboratory results and progress notes from healthcare providers.

\section{Results}

We evaluated records from 8 hospitals from the period of January 1, 2008, through April 30, 2019, for C. auris isolates. Cultures were obtained as part of routine clinical practice. A total of 28 patients from 5 hospitals had $\geq 1$ positive culture for $C$. auris during the study period. We included 28 C. auris isolates in this study (the first isolate from our health system was collected in May 2018). Members of the cohort had a median age of 70 years (interquartile range 62-78 years), and most (20 [71\%]) patients were men. Most (26 [93\%]) patients were admitted from a skilled nursing facility; 1 patient was transferred from another hospital, and 1 was admitted from the community. Nine $(75 \%)$ patients required chronic mechanical ventilation, and $6(21 \%)$ were receiving hemodialysis through a central line. Most isolates were cultured from blood (12 [43\%]) or urine (10 [36\%]). The median time from admission to collection of the first culture positive for $C$. auris was 0.14 days (interquartile range $0-0.88$ days). The average hospital stay for inpatients was 12 days. Thirteen patients $(46 \%)$ were admitted to an ICU; the average ICU stay was 3 days.

MICs for the 28 C. auris isolates (Table 1) showed that $4(14 \%)$ were resistant to fluconazole, $1(3.6 \%)$ was resistant to amphotericin B, and 1 (3.6\%) was resistant to echinocandins, according to tentative $C$. auris breakpoints published by CDC (22). One isolate was resistant to fluconazole, amphotericin B, and echinocandins. This isolate was from a patient who was considered to be colonized with $C$. auris in the urine and did not receive antifungal therapy.

Twelve patients $(43 \%)$ were treated as inpatients for $C$. auris infections (Table 2). Of those patients who

\begin{tabular}{|c|c|c|c|c|c|c|c|c|c|c|c|c|c|c|}
\hline \multirow[b]{2}{*}{ Antifungal drug } & \multicolumn{14}{|c|}{$\mathrm{MIC}, \mu \mathrm{g} / \mathrm{mL}$} \\
\hline & 0.015 & 0.03 & 0.06 & 0.12 & 0.25 & 0.5 & 1 & 2 & 4 & 8 & 64 & 128 & 256 & $>256$ \\
\hline Anidulafungin & & & 3.6 & 64.3 & 28.6 & & & & & 3.6 & & & & \\
\hline Caspofungin & & 3.6 & 25.0 & 42.9 & 25.0 & & & 3.6 & & & & & & \\
\hline Micafungin & & & 50.0 & 39.3 & 3.6 & 3.6 & & & & 3.6 & & & & \\
\hline Fluconazole & & & & & & & & 39.3 & 35.7 & 10.7 & 3.6 & 3.6 & 3.6 & 3.6 \\
\hline Itraconazole & & 7.1 & 32.1 & 39.3 & 17.9 & 3.6 & & & & & & & & \\
\hline Posaconazole & 10.7 & 50.0 & 25.0 & 14.3 & & & & & & & & & & \\
\hline Voriconazole & 42.9 & 21.4 & 21.4 & & & 3.6 & & & 10.7 & & & & & \\
\hline Amphotericin B & & & 10.7 & & & & 82.1 & 3.6 & & & & & & \\
\hline Flucytosine & & & 17.9 & 71.4 & 10.7 & & & & & & & & & \\
\hline
\end{tabular}


were not treated with antifungal therapy, 2 were evaluated in the emergency department and were discharged back to their skilled nursing facility in stable condition before their blood cultures results were available, 3 patients died before culture results were available, and 11 were considered to be colonized with $C$. auris. Of those patients who were treated for C. auris infections, most were found to have a central

Table 2. Demographic and clinical characteristics of patients treated for Candida auris infections in a multisite health system, Illinois, USA * $^{*}$

\begin{tabular}{|c|c|c|c|c|c|c|}
\hline $\begin{array}{l}\text { Patient } \\
\text { age, y/sex }\end{array}$ & $\begin{array}{l}\text { Culture source } \\
\text { (infection type) }\end{array}$ & $\begin{array}{l}\text { Empiric } \\
\text { treatment }\end{array}$ & $\begin{array}{l}\text { Definitive } \\
\text { treatment }\end{array}$ & $\begin{array}{c}\text { Treatment } \\
\text { duration }\end{array}$ & Outcome & Comments \\
\hline $83 / \mathrm{M}$ & Urine (CA-UTI) & $\begin{array}{l}\text { Micafungin } \\
100 \mathrm{mg} \text { IV } \\
\text { every } 24 \mathrm{~h}\end{array}$ & $\begin{array}{l}\text { Micafungin } \\
100 \mathrm{mg} \text { IV } \\
\text { every } 24 \mathrm{~h}\end{array}$ & $5 d$ & $\begin{array}{l}\text { Clinical } \\
\text { success }\end{array}$ & $\begin{array}{l}\text { Trach to vent patient with dementia. Urine culture } \\
\text { earlier in admission showed } 10,000-50,000 \text { CFU C. } \\
\text { auris, but thought to be colonization and was not } \\
\text { treated. Repeat urine culture showed }>100,000 \\
\text { CFU C. auris, and patient was treated. }\end{array}$ \\
\hline $56 / \mathrm{M}$ & Blood (CLABSI) & $\begin{array}{l}\text { Micafungin } \\
100 \mathrm{mg} \text { IV } \\
\text { every } 24 \mathrm{~h}\end{array}$ & $\begin{array}{l}\text { Fluconazole } \\
200 \text { mg per } \\
\text { PEG every }\end{array}$ & $15 d$ & $\begin{array}{l}\text { Clinical } \\
\text { success }\end{array}$ & $\begin{array}{c}\text { Trach to vent patient with ESRD on HD with } \\
\text { tunneled catheter, also had a PICC. Both lines were } \\
\text { removed. }\end{array}$ \\
\hline
\end{tabular}

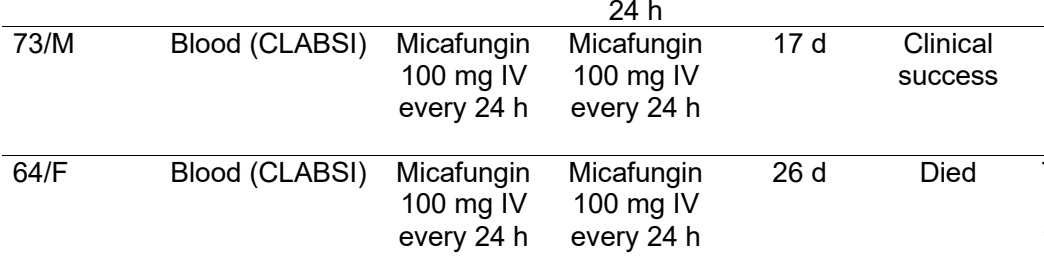

Trach to vent patient with ESRD on HD with tunneled catheter, chronic osteomyelitis of the coccyx. C. auris from culture of HD line at SNF. Tunneled catheter removed.

Trach to vent patient with ESRD on HD with chest port and PICC for TPN. Lines removed. $42 \mathrm{~d}$ of therapy planned; patient readmitted for presumed septic shock and died on day 26 after being switched to comfort care. No growth of any organisms in cultures on readmission.

\begin{tabular}{|c|c|c|c|c|c|c|}
\hline 61/M & Catheter tip & $\begin{array}{l}\text { Micafungin } \\
100 \mathrm{mg} \mathrm{IV} \\
\text { every } 24 \mathrm{~h}\end{array}$ & $\begin{array}{l}\text { Micafungin } \\
100 \mathrm{mg} \mathrm{IV} \\
\text { every } 24 \mathrm{~h}\end{array}$ & $21 \mathrm{~d}$ & $\begin{array}{l}\text { Clinical } \\
\text { success }\end{array}$ & $\begin{array}{l}\text { Trach patient with ESRD on HD with tunneled } \\
\text { catheter admitted for fungemia. Started on } \\
\text { micafungin before admission. Line removed. Azole } \\
\text { not used because of concomitant amiodarone. }\end{array}$ \\
\hline 74/M & Urine (CA-UTI) & $\begin{array}{l}\text { Micafungin } \\
100 \mathrm{mg} \text { IV } \\
\text { every } 24 \mathrm{~h}\end{array}$ & $\begin{array}{l}\text { Micafungin } \\
100 \mathrm{mg} \text { IV } \\
\text { every } 24 \mathrm{~h}\end{array}$ & Unknown & $\begin{array}{l}\text { Clinical } \\
\text { success }\end{array}$ & $\begin{array}{c}\text { Trach to vent patient. Patient transferred to SNF } \\
\text { before culture finalized; duration of micafungin to be } \\
\text { determined by SNF. }\end{array}$ \\
\hline $74 / F$ & Blood (CLABSI) & $\begin{array}{l}\text { Micafungin } \\
100 \mathrm{mg} \text { IV } \\
\text { every } 24 \mathrm{~h}\end{array}$ & $\begin{array}{l}\text { Fluconazole } \\
400 \mathrm{mg} \mathrm{PO} \\
\text { every } 24 \mathrm{~h}\end{array}$ & $21 \mathrm{~d}$ & $\begin{array}{l}\text { Clinical } \\
\text { success }\end{array}$ & $\begin{array}{l}\text { SNF patient on chronic TPN for enterocutaneous } \\
\text { fistulas, history of line infections and infective } \\
\text { endocarditis. Persistently fungemic for } 4 \mathrm{~d} \text { until } \\
\text { tunneled central line was removed. }\end{array}$ \\
\hline $50 / F$ & $\begin{array}{l}\text { Abdominal } \\
\text { wound }\end{array}$ & $\begin{array}{l}\text { Micafungin } \\
100 \mathrm{mg} \text { IV } \\
\text { every } 24 \mathrm{~h}\end{array}$ & $\begin{array}{l}\text { Micafungin } \\
100 \mathrm{mg} \text { IV } \\
\text { every } 24 \mathrm{~h}\end{array}$ & $10 \mathrm{~d}$ & $\begin{array}{l}\text { Clinical } \\
\text { success }\end{array}$ & $\begin{array}{c}\text { Patient with obesity, diabetes, and chronic } \\
\text { abdominal/groin ulcers hospitalized for DKA; } \\
\text { receives wound care at home. Ulcers underwent } \\
\text { debridement; } C \text {. auris, CoNS, and Corynebacterium } \\
\text { grew from operative cultures. }\end{array}$ \\
\hline 78/M & Blood & $\begin{array}{l}\text { Fluconazole } \\
400 \mathrm{mg} \text { IV } \\
\text { every } 24 \mathrm{~h}\end{array}$ & $\begin{array}{l}\text { Itraconazole } \\
200 \text { mg per } \\
\text { PEG every } \\
24 \mathrm{~h}\end{array}$ & $14 \mathrm{~d}$ & $\begin{array}{l}\text { Clinical } \\
\text { success }\end{array}$ & $\begin{array}{l}\text { Trach to vent after cardiac arrest, midline POA for } \\
\text { hypotension and hypoxia. Midline thought to be } \\
\text { source. Discharged to hospice, but continued } \\
\text { antifungal therapy. Lost to follow-up. }\end{array}$ \\
\hline 79/M & Blood (CLABSI) & $\begin{array}{l}\text { Micafungin } \\
100 \mathrm{mg} \text { IV } \\
\text { every } 24 \mathrm{~h}\end{array}$ & $\begin{array}{l}\text { Micafungin } \\
100 \mathrm{mg} \text { IV } \\
\text { every } 24 \mathrm{~h}\end{array}$ & $5 d$ & Died & $\begin{array}{l}\text { Trach, ESRD on HD with tunneled catheter. Blood } \\
\text { culture also showed growth of Proteus mirabilis. } \\
\text { Died from septic shock after switching to comfort } \\
\text { care. Repeat blood cultures showed no growth. }\end{array}$ \\
\hline $78 / F$ & $\begin{array}{l}\text { Hip synovial } \\
\text { fluid }\end{array}$ & $\begin{array}{l}\text { Micafungin } \\
100 \mathrm{mg} \text { IV } \\
\text { every } 24 \mathrm{~h}\end{array}$ & $\begin{array}{l}\text { Micafungin } \\
100 \mathrm{mg} \text { IV } \\
\text { every } 24 \mathrm{~h}\end{array}$ & $6 \mathrm{~d}$ & $\begin{array}{l}\text { Clinical } \\
\text { success }\end{array}$ & $\begin{array}{l}\text { ESRD on HD with tunneled catheter, DM, prosthetic } \\
\text { mitral valve, treated for drainage from hip after hip } \\
\text { replacement } 3 \text { mo prior, had onset of septic shock } \\
\text { after I\&D procedure. } C \text { auris isolated from hip } \\
\text { aspirate. Antifungal treatment stopped after } 6 \mathrm{~d} \\
\text { because } C \text {. auris was a suspected contaminant. } \\
\text { Died in hospital }>30 \mathrm{~d} \text { after } C \text { auris isolation. }\end{array}$ \\
\hline $82 / \mathrm{M}$ & Blood (CLABSI) & $\begin{array}{l}\text { Micafungin } \\
100 \mathrm{mg} \text { IV } \\
\text { every } 24 \mathrm{~h}\end{array}$ & $\begin{array}{l}\text { Micafungin } \\
100 \mathrm{mg} \text { IV } \\
\text { every } 24 \mathrm{~h}\end{array}$ & $14 \mathrm{~d}$ & $\begin{array}{l}\text { Clinical } \\
\text { success }\end{array}$ & $\begin{array}{c}\text { Patient with functional quadriplegia after CVA. } \\
\text { Trach, PEG, PICC, and chronic foley catheter POA. } \\
\text { PICC removed. }\end{array}$ \\
\hline
\end{tabular}

${ }^{*} \mathrm{CA}-\mathrm{UTI}$, catheter-associated urinary tract infection; CFU, colony forming units; CLABSI, catheter-associated urinary tract infection; CoNS, coagulase negative Staphylococci; CVA, cerebral vascular accident; DKA, diabetic ketoacidosis; DM, diabetes mellitus; ESRD, end-stage renal disease; HD, hemodialysis; I\&D, incision and debridement; PEG, percutaneous endoscopic gastrostomy; PICC, peripherally inserted central catheter; POA, present on admission; SNF, skilled nursing facility; TPN, total parenteral nutrition; trach, tracheotomy; vent, ventilator. 
line-associated bloodstream infection (CLABSI) (7 [59\%]), whereas others were treated for catheter-associated urinary tract infection (2 [17\%]), skin and skin structure infection (2 [17\%]), and other bloodstream infection (BSI) (1 [8\%]). All patients who were treated for $C$. auris infections were under the care of a physician specialized in infectious diseases.

Patients were empirically treated with micafungin (11 [92\%]) or fluconazole (1 [8\%]). Of those patients empirically treated with micafungin, most were being treated for CLABSI (7 [64\%]), followed by catheter-associated urinary tract infection $(2[18 \%])$ and skin and skin structure infection (2 [18\%]). For definitive treatment, patients received micafungin (9 $[75 \%])$, fluconazole $(2[17 \%])$, or itraconazole (1 [8\%]). Of those patients who received an azole as definitive treatment, all were being treated for BSIs. Treatment duration ranged from 5 to 26 days (mean 14 days). The only adverse event noted was an increase in aspartate aminotransferase from 25 to $91 \mathrm{U} / \mathrm{L}$ in 1 patient being treated with fluconazole. Fluconazole was continued, and the patient was discharged on fluconazole to complete their treatment course.

Ten $(83 \%)$ patients met criteria for clinical success. No patients were found to have C. auris recurrence or infection-related readmission within 30 days of first positive culture. Two (17\%) patients died within 30 days of first positive culture; both were being treated for CLABSIs.

\section{Discussion}

We report patient characteristics and microbiologic data for 28 patients with $\geq 1$ positive culture for $C$. auris. We also describe the clinical outcomes for 12 patients treated for C. auris infections. Our observed mortality rate of $17 \%$ is lower than previously reported worldwide. A meta-analysis of 742 patients from 16 countries found an all-cause mortality rate of 30\% (13). Our lower mortality rate might be a result of empirically selecting echinocandin therapy, to which $>95 \%$ of our isolates were susceptible. Empiric selection of fluconazole for treatment of $C$. auris BSI was recently described in a case series in India (19). C. auris is often resistant to fluconazole, and lack of effective empiric therapy can result in poor outcomes. Because echinocandins are commonly empirically selected for treatment of candidemia in the United States, our lower mortality rates might be attributable to lower MICs in our geographic region. For instance, investigators of an outbreak in New York, New York, found a $45 \%$ mortality rate among 51 patients with BSIs at 90 days (10). The mortality rate in our study was $25 \%$ among patients with BSI. A separate case series from Brooklyn,
New York, reported a 22\% in-hospital mortality rate among a cohort of 9 patients with BSIs, which is similar to our findings (17). Small sample size and variations in underlying conditions might confound mortality rate comparisons, although another possible explanation for the lower mortality rate in our study is differences in antifungal resistance.

Previous reports of $C$. auris collections have noted substantially higher rates of antifungal resistance than what was observed in our cohort. In a grouping of 54 isolates from 5 countries, 93\% were resistant to fluconazole, $35 \%$ to amphotericin $\mathrm{B}$, and $7 \%$ to echinocandins (16). The first isolates from the United States demonstrated a similar pattern; $86 \%$ were resistant to fluconazole, $43 \%$ to amphotericin B, and 3\% to echinocandins (18). An even higher rate of fluconazole resistance of $98 \%$ was noted in New York, New York (10). Drug-resistance mechanisms are genetically encoded, and some resistance mutations are linked to specific geographic clades (16). An epidemiologic investigation of 133 C. auris isolates from the United States showed that these isolates were genetically related to 1 of 4 major clades (from South America, Africa, East Asia, and South Asia) (20). Previous isolates from Illinois were found to be from the South America clade $(15,20)$. The molecular epidemiology of the isolates we identified was beyond the scope of this investigation because the results of genomic sequencing were not shared with clinicians or laboratory personnel within our health system. Further research would be needed to determine whether these isolates are genetically distinct from clades previously noted to be present in the United States.

Most patients in this study were successfully treated with an echinocandin, which is consistent with treatment recommendations from the CDC (22). The 2 members of our cohort who died within 30 days of first positive culture attained microbiologic eradication with echinocandin therapy before they died. Three patients received azole antifungals as empiric or definitive treatment, and all 3 met our criteria for clinical success. However, treatment failures with fluconazole have been reported despite in vitro susceptibility $(8,21)$. Echinocandin-resistant $C$. auris, possibly induced by antifungal pressure, has also been reported in the United States and in other countries $(16,21)$. Patients with persistent or recurrent C. auris infections might require repeat susceptibility testing.

Several antifungal agents currently in development have activity against $C$. auris, including SCY078 , the first orally bioavailable $1,3-\beta$-D-glucan synthesis inhibitor; VT-1598, a tetrazole-based lanosterol 14a-demethylase inhibitor; APX001, which interrupts 
glycosylphosphatidylinositol biosynthesis by inhibiting the fungal enzyme Gwt1; and CD101, an echinocandin that can be administered once weekly (12). In the future, these agents might become treatment options for $C$. auris infections, including those caused by isolates resistant to conventional therapies.

Most patients in our study who required treatment for $C$. auris were previously exposed to skilled nursing facilities and had multiple risk factors for invasive Candida infections, including central venous and urinary catheters (12). No patients were thought to have acquired C. auris infection during their hospital admission. The possibility of transmission within hospitals is a concern, given that $C$. auris has been shown to colonize the skin, persist on surfaces in the healthcare environment, and cause healthcare-associated outbreaks $(10,21)$. Contact precautions, hand hygiene, and environmental cleaning and disinfection are essential to preventing the spread of $C$. auris, and these infection control practices are used within our health system (22).

Our study is limited by a small sample size and the inherent limitations of a retrospective, observational case series. In addition, evaluation of clinical outcomes at 30 days from the first positive culture prevented us from capturing any adverse outcomes that might have occurred after 30 days. However, our data provides insight on patient exposures. Most of these patients came from skilled nursing facilities. Targeted infection prevention and antimicrobial stewardship measures within these facilities might help to reduce the emergence and progression of resistance of this organism. Our experiences with $C$. auris are notable for favorable clinical outcomes and low rates of antifungal resistance. However, the development of resistance remains a concern, and patient response to treatment should be monitored closely.

\section{Acknowledgments}

We thank Eric Beck for his assistance with describing our laboratory's identification and susceptibility testing protocols.

\section{About the Author}

Dr. Arensman is a postgraduate year 2 infectious diseases pharmacy resident at Advocate Lutheran General Hospital in Park Ridge, Illinois. Her primary research interests are treatment of bloodstream infections and antimicrobial stewardship.

\section{References}

1. Satoh K, Makimura K, Hasumi Y, Nishiyama Y, Uchida K, Yamaguchi H. Candida auris sp. nov., a novel ascomycetous yeast isolated from the external ear canal of an inpatient in a
Japanese hospital. Microbiol Immunol. 2009;53:41-4. https://doi.org/10.1111/j.1348-0421.2008.00083.x

2. Lee WG, Shin JH, Uh Y, Kang MG, Kim SH, Park KH, et al. First three reported cases of nosocomial fungemia caused by Candida auris. J Clin Microbiol. 2011;49:3139-42. https://doi.org/10.1128/JCM.00319-11

3. Chowdhary A, Sharma C, Duggal S, Agarwal K, Prakash A, Singh PK, et al. New clonal strain of Candida auris, Delhi, India. Emerg Infect Dis. 2013;19:1670-3. https://doi.org/10.3201/eid1910.130393

4. Magobo RE, Corcoran C, Seetharam S, Govender NP. Candida auris-associated candidemia, South Africa. Emerg Infect Dis. 2014;20:1250-1. https:/ / doi.org/10.3201/ eid2007.131765

5. Emara M, Ahmad S, Khan Z, Joseph L, Al-Obaid I, Purohit P, et al. Candida auris candidemia in Kuwait, 2014. Emerg Infect Dis. 2015;21:1091-2. https:// doi.org/10.3201/ eid2106.150270

6. Schelenz S, Hagen F, Rhodes JL, Abdolrasouli A, Chowdhary A, Hall A, et al. First hospital outbreak of the globally emerging Candida auris in a European hospital. Antimicrob Resist Infect Control. 2016;5:35. https:/ / doi.org/ 10.1186/s13756-016-0132-5

7. Calvo B, Melo AS, Perozo-Mena A, Hernandez M, Francisco EC, Hagen F, et al. First report of Candida auris in America: clinical and microbiological aspects of 18 episodes of candidemia. J Infect. 2016;73:369-74. https:/ / doi.org/10.1016/j.jinf.2016.07.008

8. Vallabhaneni S, Kallen A, Tsay S, Chow N, Welsh R, Kerins J, et al. Investigation of the first seven reported cases of Candida auris, a globally emerging invasive, multidrug-resistant fungus - United States, May 2013-August 2016. MMWR Morb Mortal Wkly Rep. 2016;65:1234-7. https:/ / doi.org/ 10.15585/mmwr.mm6544e1

9. Heath CH, Dyer JR, Pang S, Coombs GW, Gardam DJ. Candida auris sternal osteomyelitis in a man from Kenya visiting Australia, 2015. Emerg Infect Dis. 2019;25:192-4. https://doi.org/10.3201/eid2501.181321

10. Adams E, Quinn M, Tsay S, Poirot E, Chaturvedi S, Southwick K, et al.; Candida auris Investigation Workgroup. Candida auris in healthcare facilities, New York, USA, 2013-2017. Emerg Infect Dis. 2018;24:1816-24. https://doi.org/10.3201/eid2410.180649

11. Sarma S, Upadhyay S. Current perspective on emergence, diagnosis and drug resistance in Candida auris. Infect Drug Resist. 2017;10:155-65. https:/ / doi.org/10.2147/IDR.S116229

12. Lone SA, Ahmad A. Candida auris - the growing menace to global health. Mycoses. 2019;62:620-37. https://doi.org/ 10.1111/myc.12904

13. Osei Sekyere J. Candida auris: a systematic review and meta-analysis of current updates on an emerging multidrugresistant pathogen. MicrobiologyOpen. 2018;7:e00578. https:/ / doi.org/ 10.1002/mbo3.578

14. Kerins JL, Tang AS, Forsberg K, Jegede O, Ealy M, Pacilli M, et al. Rapid emergence of Candida auris in the Chicago region. Open Forum Infect Dis. 2018;5(S1):S28. https:/ / doi.org/ 10.1093/ofid/ofy209.064

15. Hayden MK, Dangana TE, Yelin RD, Schoeny M, Bell PB, Stanley M, et al. Prevalence of Candida auris at body sites, characterization of skin microbiota, and relation of chlorhexidine gluconate (CHG) skin concentration to C. auris detection among patients at a high-prevalence ventilator-capable skilled nursing facility (vSNF) with established CHG bathing. Open Forum Infect Dis. 2019;6(S2):S25-6. https:// doi.org/10.1093/ofid/ ofz359.056 
16. Lockhart SR, Etienne KA, Vallabhaneni S, Farooqi J, Chowdhary A, Govender NP, et al. Simultaneous emergence of multidrug-resistant Candida auris on 3 continents confirmed by whole-genome sequencing and epidemiological analyses. Clin Infect Dis. 2017;64:134-40. https://doi.org/10.1093/cid/ciw691

17. Park JY, Bradley N, Brooks S, Burney S, Wassner C. Management of patients with Candida auris fungemia at community hospital, Brooklyn, New York, USA, 2016-2018. Emerg Infect Dis. 2019;25:601-2. https://doi.org/10.3201/ eid2503.180927

18. Tsay S, Welsh RM, Adams EH, Chow NA, Gade L, Berkow EL, et al. Ongoing transmission of Candida auris in health care facilities - United States, June 2016-May 2017. MMWR Morb Mortal Wkly Rep. 2017;66:514-5. https://doi.org/10.15585/mmwr.mm6619a7

19. Bajpai V, Govindaswamy A, Sagar S, Kumar S, Garg P, Xess I, et al. Multidrug-resistant fungemia in critical care units: experience from a tertiary care hospital in India. Microb Drug Resist. 2019;mdr.2019.0021 [Epub ahead of print]. https://doi.org/ 10.1089/ mdr.2019.0021
20. Chow NA, Gade L, Tsay SV, Forsberg K, Greenko JA, Southwick KL, et al.; US Candida auris Investigation Team. Multiple introductions and subsequent transmission of multidrug-resistant Candida auris in the USA: a molecular epidemiological survey. Lancet Infect Dis. 2018;18:1377-84 https:/ / doi.org/10.1016/S1473-3099(18)30597-8

21. Biagi MJ, Wiederhold NP, Gibas C, Wickes BL, Lozano V, Bleasdale SC, et al. Development of high-level echinocandin resistance in a patient with recurrent Candida auris candidemia secondary to chronic candiduria. Open Forum Infect Dis. 2019;6:ofz262. https://doi.org/10.1093/ofid/ ofz262

22. Tsay S, Kallen A, Jackson BR, Chiller TM, Vallabhaneni S. Approach to the investigation and management of patients with Candida auris, an emerging multidrug-resistant yeast. Clin Infect Dis. 2018;66:306-11. https:/ / doi.org/10.1093/ cid/cix744

Address for correspondence: Jennifer Dela Pena, Advocate Lutheran General Hospital, 1775 Dempster St, Park Ridge, IL 60068 USA; email: jennifer.delapena@advocatehealth.com

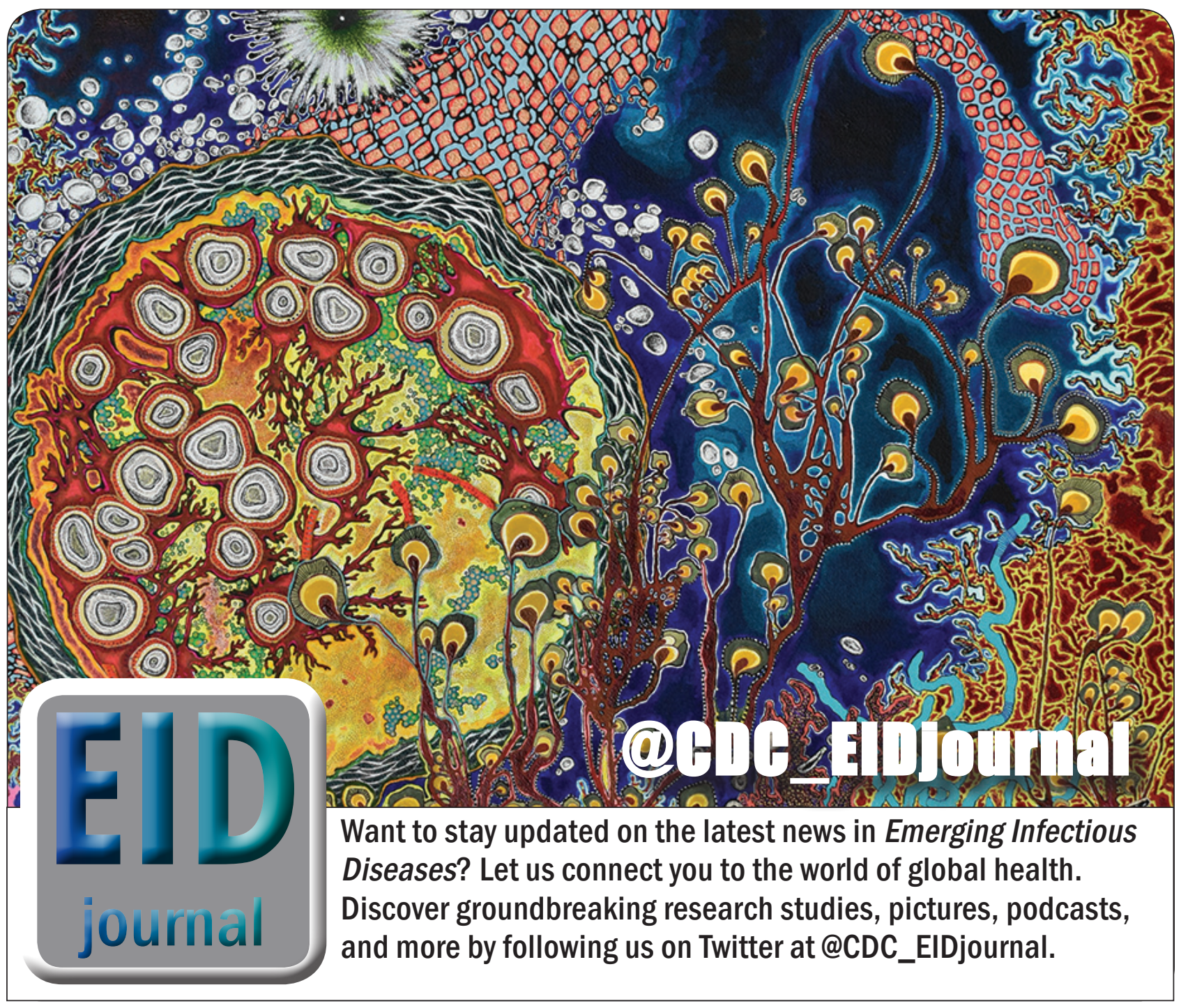

